\title{
Failure of Ischemic Neuroprotection by Potentiators of Gamma-aminobutyric Acid
}

\author{
Ken Madden, MD, PhD, Department of Neurology, Marshfield Clinic, Marshfield, Wisconsin \\ Wayne Clark, MD, Oregon Health Sciences University, Portland, Oregon \\ Nicola Lessov, MD, Oregon Health Sciences University, Portland, Oregon
}

\section{ABSTRACT}

\section{INTRODUCTION}

Potentiators of inhibitory neurotransmission may provide a neuroprotective effect on cerebral tissue exposed to ischemia, without inducing toxic side effects. Topiramate and vigabatrin enhance the action of gamma-aminobutyric acid (GABA), and each has side effect profiles known to be well tolerated through their clinical use as anticonvulsant medications. We assessed the potential benefit through GABA activation by these drugs on infarct size and functional recovery following focal cerebral ischemia in mice.

\section{METHODS}

Silicon-coated suture was advanced through the internal carotid artery of 89 halothane-anesthetized mice to temporarily occlude the right middle cerebral artery for either 45 minutes (topiramate), or 120 minutes (vigabatrin). Animals were treated either at the time of reperfusion with topiramate $(100 \mathrm{mg} / \mathrm{kg}, 40 \mathrm{mg} / \mathrm{kg}$, or saline control), or two hours before arterial occlusion with vigabatrin, (1000 mg/kg, $500 \mathrm{mg} / \mathrm{kg}$, or saline control). Neurological outcome was measured 24 hours after ischemia using a 28-point functional examination score. Infarct volume was estimated by summing area maps of stained slices of infarcted hemispheres.

RESULTS

Functional examination scores at 24 hours were similar between the high dose topiramate group, the low dose topiramate group, and the control group. Similarly, no differences were noted between examination scores of high dose vigabatrin, low dose vigabatrin, and control. Consistent sized right hemisphere infarcts were noted within each group on histological examination. Mean infarct volumes did not differ between groups treated with high dose topiramate, low dose topiramate, or control. Infarct volumes of animals treated with saline control were slightly larger than that of high dose vigabatrin and low dose vigabatrin groups, but the difference did not reach significance.

CONCLUSION

Treatment with these two potentiators of GABA did not result in significant differences in outcome following focal cerebral ischemia, by either functional or histological measures. These results do not support a substantial neuroprotective role of GABA following ischemia in this mouse suture model.

RECEIVED: JANUARY 10,2003

\section{REPRINT REQUESTS:}

Ken Madden, MD, PhD

Department of Neurosciences

Marshfield Clinic

1000 North Oak Avenue (4F2)

Marshfield, WI 54449

Telephone: $715-389-3225$

Fax: 715-387-5727

Email: madden.kenneth@marshfieldclinic.org

REVISED AND ACCEPTED: MARCH 4, 2003

KEYWORDS:

Cerebral ischemia; GABA; Animal model; Neuroprotection

GRANT SUPPORT:

Vascular Research Grant, Marshfield Clinic Research Foundation 


\section{INTRODUCTION}

Stroke remains the third-leading cause of mortality in North America and the leading cause of morbidity. Recent advances in the use of drug-induced thrombolysis and mechanical obliteration of occlusive arterial disease have resulted in improvement in some patients with stroke. However, patients eligible for this type of therapy represent a small proportion of patients presenting with acute cerebral ischemia, ${ }^{1}$ primarily because revascularization must occur within a short period of time from the onset of ischemia. Reperfusion delayed longer than 3 to 6 hours promises little in the way of clinical benefit, ${ }^{2}$ predominantly because neurons have already been irreversibly damaged within the ischemic tissue bed. Drugs that protect neurons exposed to such conditions offer the potential to expand the therapeutic window for revascularization techniques, or simply allow greater survival of affected neurons until spontaneous reperfusion occurs. Unfortunately, clinical trials have yet to identify a drug that provides effective neuroprotection for patients suffering from stroke. ${ }^{3}$ Notably, potential benefit of some of these drugs may have been masked by toxic side effects that only became apparent through their use during clinical trials.

Ischemic injury to cerebral tissue triggers complex biochemical cascades that ultimately lead to neuronal death.

Excessive excitatory neurotransmitter release occurs during this process, stimulating "excitotoxic" conditions that hasten depletion of oxygen and nutrients. Gamma-aminobutyric acid (GABA) is the most abundant inhibitory neurotransmitter of human cerebrum. Pharmacological activators of GABA represent a physiological means to inhibit cellular activity within the brain, possibly blunting ischemic injury. Previous work in this laboratory demonstrated that an agonist of GABA improved tolerance of rabbit spinal cord to ischemic injury, while an antagonist of GABA worsened tolerance. ${ }^{4}$ GABA activators are currently used clinically as anti-spasmodic or anticonvulsant medications, and they have a generally well-tolerated side-effect spectrum.

Topiramate is a recently developed anticonvulsant approved for human use. Topiramate raises brain levels of GABA through inhibition of its reuptake, and potentiates its inhibitory neurotransmitter effect by increasing the frequency of GABA interactions with its receptor. ${ }^{5}$ During clinical trials using doses effective for seizure prevention, $28 \%$ of patients discontinued the medication citing side effects, but only $1.3 \%$ discontinued for a reason potentially concerning for short-term use (excess sedation). Vigabatrin is an anticonvulsant drug currently marketed in Europe. This drug irreversibly inhibits GABA-transaminase, resulting in increased brain GABA concentration. ${ }^{6}$ It is rapidly absorbed by either oral or intraperitoneal administration and has been widely investigated as an experimental anticonvulsant in animals. The side effect profile in humans with doses effective for epilepsy ( 50 to $100 \mathrm{mg} / \mathrm{kg}$ ) is considered mild and quite acceptable (sedation, confusion, fatigue). A mouse model of focal cerebral ischemia was utilized to explore the potential benefit of topiramate and vigabatrin as neuroprotectants that would likely be well tolerated in clinical use.

\section{MATERIALS AND METHODS}

The Marshfield Clinic Research Foundation provided funding for this study. All animal procedures were approved by the Oregon Health Sciences University Institutional Review Board and are in accordance with guidelines published by the National Institute of Health for animal use. Experimental techniques differed slightly between topiramate and vigabatrin groups, conforming to the evolution of the animal model in this laboratory over time.

\section{CNS Ischemia Model}

Topiramate: Forty-four male C57bl/6 mice weighing approximately 25 to 30 grams were used in this study. The animals were anesthetized via inhalation mask with $1.25 \%$ to $1.5 \%$ halothane/oxygen. A skin incision in the right temporoparietal area was made, the temporalis muscle was retracted, and a microtip of the laser-Doppler probe (Periflux System 5000) was glued to the skull with cyanoacrylate adhesive (Krazy Glue). Continuous cerebral blood flow was recorded throughout the experiment.

A rectal temperature probe was introduced, and a heating pad maintained body temperature at $37 \pm 0.5^{\circ} \mathrm{C}$. Under a dissecting microscope, the right carotid bifurcation was exposed, and the external carotid artery was coagulated distal to the bifurcation. After temporary ligature of the common carotid artery, an 8-0 silicone-coated nylon filament was inserted through the external carotid artery stump and gently advanced ( 8 to $9 \mathrm{~mm}$ ) to occlude the origin of the ipsilateral middle cerebral artery, following a modification of the method originally reported by Koizumi et al. ${ }^{7}$ Successful middle cerebral artery filament occlusion (defined as $80 \%$ or greater reduction of baseline blood flow) was confirmed by laser Doppler. For the subsequent 45 minutes, the animal remained untouched under constant conditions. At the end of the period, the filament was withdrawn, the external carotid artery stump was tied, the common carotid artery was untied, and reperfusion was confirmed by laser Doppler (defined as $85 \%$ or greater restoration of baseline blood flow). The animals were then permitted to recover from anesthesia on a warming pad (at $24^{\circ} \mathrm{C}$ ) and sheltered from drafts.

Vigabatrin: Forty-five mice were divided into three experimental groups. Animal care and surgical techniques were similar to the topiramate group. Laser Doppler techniques were not employed for these experimental groups.

\section{Drug Administration}

Topiramate (lot \# 99TOP024A) was supplied by R.W. Johnson Pharmaceutical Research Institute. The control vehicle was sterile $0.9 \%$ sodium chloride $(\mathrm{NaCl})$. Upon arrival, topiramate was stored at room temperature inside a desiccator, protected from light. The experimental mice were divided randomly into 3 groups. Animals received control, 
topiramate $(40 \mathrm{mg} / \mathrm{kg}$ in $0.1 \mathrm{ml}$ volume), or topiramate (100 $\mathrm{mg} / \mathrm{kg}$ in $0.1 \mathrm{ml}$ volume) by intraperitoneal injection. Drug or vehicle was given just after the 45 minute period of cerebral ischemia was completed (approximately five minutes post-reperfusion).

Vigabatrin was suppled by Marion Merrell Dow, Incorporated. This drug was handled in a similar manner as topiramate. The vehicle was again $0.9 \% \mathrm{NaCl}$. Animals were treated 2 hours before arterial occlusion with a single intraperitoneal dose of saline or vigabatrin, either 1000 $\mathrm{mg} / \mathrm{kg}$ or $500 \mathrm{mg} / \mathrm{kg}$. Injection volumes ranged to $0.3 \mathrm{ml}$.

\section{Neurological Function and Infarct Volume}

At 24 hours, the animals were scored for neurological deficit by a 28 -point focal scoring system. ${ }^{8}$ Scores are assigned to each animal (from a 0 to 4 point scale) for each of seven functional tests: gait, body symmetry, climbing, turning behavior, front leg extension, compulsory circling, sensory response. Two investigators independently scored the animals, and scores were averaged. The animals were anesthetized (Isoflurane) and quickly sacrificed. Whole brain tissue from the topiramate group was collected and immediately placed in a warm bed of $1.5 \%$ agarose. The tissue was then sectioned into six $1 \mathrm{~mm}$ sections using a Stoelting tissue slicer. Sections were placed directly into well plates containing $2 \%$ tetrazolium chloride (TTC) and allowed to develop in a $37^{\circ} \mathrm{C}$ water bath for 30 minutes. Each tissue slice was placed in $10 \%$ formalin for 24 hour fixation. Animals in the vigabatrin group were sacrificed at

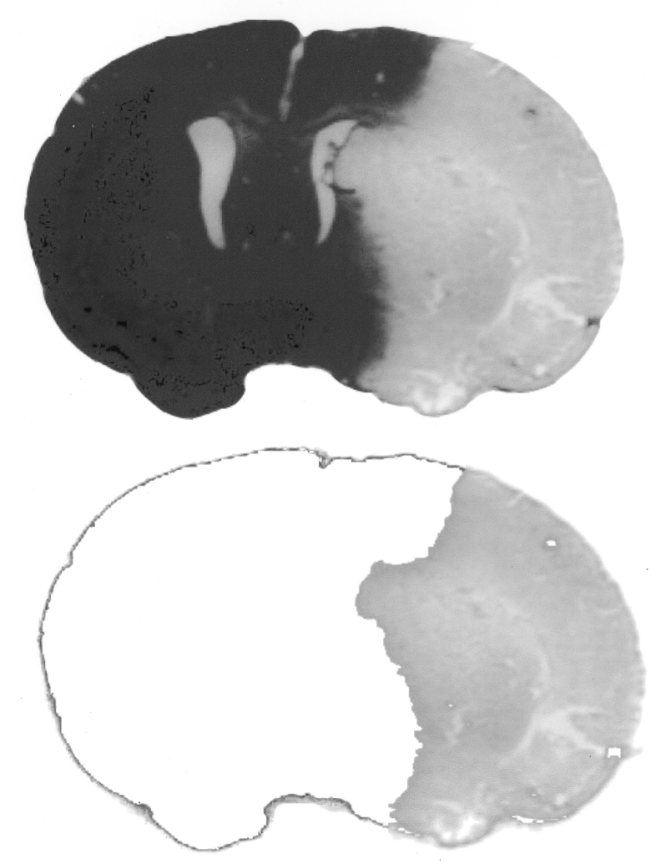

Figure 1. Representative slice of mouse brain exposed to right middle cerebral artery ischemia. Infarcted tissue absorbs stain (Luxol fast blue and Cresyl violet) less readily and allows image manipulation to extract the injured region (lower panel). Area of infarction for each brain section can then be calculated.
48 hours and brains were perfusion-fixed with $4 \%$ formalin. Brains were harvested and stained with Luxol fast blue and Cresyl violet. This tissue was sliced in 50-micrometer sections, and every 10 th slice was selected for analysis. Slice faces from all experimental groups were subsequently scanned, and image files were imported into Adobe PhotoShop. For all brain slices, the National Institutes of Health Image program was used to measure the cerebral hemisphere area and the area of ischemic change, as reflected by stain uptake differences on each slice face (figure 1). Total hemispheric volumes and volumes of ischemic injury were calculated by summing the products of each slice thickness and its measured areas (figure 2). The measured area of each 50-micrometer slice from the vigabatrin group was presumed representative of the 500-micrometer thickness of tissue surrounding it. To partially correct for effects of edema, ischemic volumes were also expressed as a percentage of its ipsilateral hemisphere volume (topiramate group only).

All results are expressed as mean \pm standard deviation (SD). ANalysis Of VAriance (ANOVA) and unpaired Student's t-test (Bonferroni-corrected) were used to assess the significance of differences in lesion volumes, using significance levels $\mathrm{p}<0.05$.

\section{RESULTS}

Topiramate: A total of 44 animals were included in the analysis after meeting all study criteria (table 1). There were no differences between groups in body weight, pre-ischemic

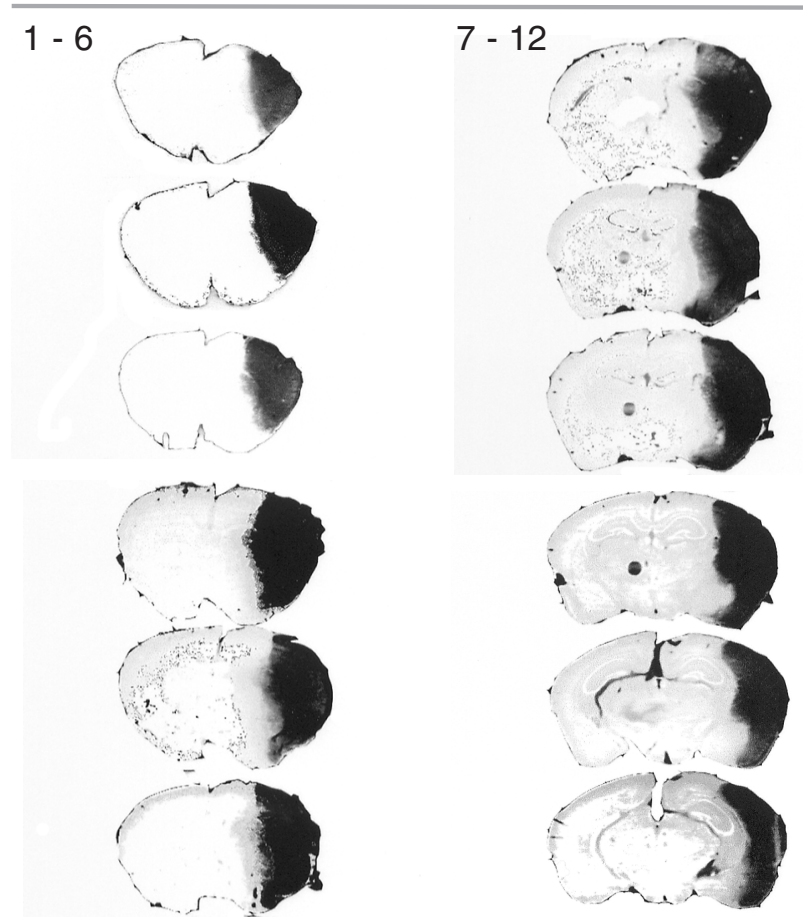

Figure 2. Complete sections of mouse brain exposed to right middle cerebral artery ischemia. Images have been manipulated to accentuate the infracted area on each slice.

Hemispheric infarct volume is the sum of all slice infarct volumes (area of infarction on each slice multiplied by its known thickness). 
Table 1. Histological and neurological exam outcome measures.

\begin{tabular}{lcccc}
\hline Animal Group & Dose & $\begin{array}{c}\text { Infarct volume } \\
(\mathbf{m m} \pm \mathbf{S . D .})\end{array}$ & $\begin{array}{c}\text { Percent infarct volume } \\
\text { Average neuro score }\end{array}$ \\
\hline Topiramate & $100 \mathrm{mg} / \mathrm{kg}$ & $11.1 \pm 2.5$ & $10.9 \pm 2.3$ & $10.5 \pm 1.6$ \\
Topiramate & $40 \mathrm{mg} / \mathrm{kg}$ & $12.0 \pm 3.1$ & $11.6 \pm 2.7$ & $10.3 \pm 1.0$ \\
Control & & $12.5 \pm 2.9$ & $12.2 \pm 2.9$ & $10.3 \pm 1.2$ \\
& & & $*$ & $12.1 \pm 6.1$ \\
Vigabatrin & $1000 \mathrm{mg} / \mathrm{kg}$ & $74.6 \pm 28.8$ & $*$ & $11.0 \pm 3.8$ \\
Vigabatrin & $500 \mathrm{mg} / \mathrm{kg}$ & $75.8 \pm 20.9$ & $*$ & $12.7 \pm 2.3$ \\
Control & & $81.7 \pm 11.2$ & &
\end{tabular}

cerebral blood flow, or post-ischemic blood flow. Infarct volumes (mean $\pm \mathrm{SD}$ ) at 24 hours were as follows: control group $(\mathrm{n}=15), 12.5 \pm 2.9 \mathrm{~mm}^{3}$; low dose group $(\mathrm{n}=15), 12.0$ $\pm 3.1 \mathrm{~mm}^{3}$; high dose group $(\mathrm{n}=14), 11.1 \pm 2.5 \mathrm{~mm}^{3}$.

Average neurological scores for the control group was $10.3 \pm$ 1.2 , compared to $10.3 \pm 1.0$ for the low dose group and 10.5 \pm 1.6 for the high dose group. There were no significant differences among lesion volumes, percent infarct volumes, or focal neurological scores.

Vigabatrin: A total of 45 mice survived and were included in the analysis (table 1). All mice developed evident signs of right hemisphere dysfunction, consistent with stroke. Fifteen animals remained in each group. Consistent sized right hemisphere infarcts were noted within each group on histological examination. Mean infarct volume of the control group (81.7 $\pm 11.2 \mathrm{~mm}^{3}$ ) was slightly larger than that of low dose vigabatrin $\left(75.8 \pm 20.9 \mathrm{~mm}^{3}\right)$ and high dose vigabatrin $(74.6 \pm 28.8$ $\mathrm{mm}^{3}$ ) groups, but the difference did not reach significance by ANOVA analysis. Functional examination scores at 48 hours were similar between the control group (12.7 \pm 2.3$)$, the low dose group $(11.0 \pm 3.8)$, and the high dose group $(12.1 \pm 6.1)$.

\section{DISCUSSION}

Experimental evidence suggests that excitatory neurotransmitters potentiate neuronal ischemic damage. Excessive stimulation of excitatory neurotransmitter receptors is damaging to neurons, and specific antagonists ${ }^{9}$ can block this neurotoxicity. Similarly, antagonists of excitatory neurotransmitters have been shown to blunt cell injury in a variety of in vitro and in vivo models of neuronal ischemia. ${ }^{10}$ A number of such drugs were assessed by clinical trial for treatment of stroke. Benefit could not be established, and side effects were substantial. ${ }^{3}$ Conversely, activators of inhibitory neurotransmitters are commonly used clinically (with acceptable side effect profiles), yet have not been extensively tested as potential neuroprotective agents.

A variety of evidence suggests increased inhibitory neurotransmitter activity may protect ischemic neurons. At the cellular level, inhibitory neurotransmitters such as GABA increase chloride conductance, effectively blunting depolarization and therefore the opening of voltage-dependent calcium channels. ${ }^{11}$ Such inhibition may also reduce the metabolic requirements of the normally perfused ${ }^{12}$ and the ischemic neuron. ${ }^{13}$ Neurons producing GABA are relatively resistant to both N-methyl-D-aspartate (NMDA)-induced neurotoxicity and ischemia. ${ }^{14-16}$ Neuronal death related to excitotoxic exposure can be ameliorated by local application of GABA potentiators. ${ }^{17}$ Regional vulnerability to ischemic injury can also be correlated with the concentration of excitatory neurotransmitters relative to that of GABA. ${ }^{18}$ The turtle brain, which is remarkably resistant to anoxic injury, produces markedly elevated GABA levels during anoxic exposures. ${ }^{19}$ In addition to metabolic effects, GABA may also elicit a potentially restorative local vasodilatory response. $^{20}$

Previous experimental work has correlated pharmacologically increased levels of GABA activity with lessened degree of neuronal ischemic injury in both brain ${ }^{21-24}$ and spinal cord. ${ }^{25}$ Other investigators, however, have not been able to demonstrate the benefit of GABAmimetic drugs in ischemia, particularly those agents relatively specific for the GABA-B receptor. ${ }^{26}$ In a rabbit model of multiple cerebral emboli, Lyden et al, have demonstrated neuroprotection by the GABA-A agonist muscimol, both alone 27 and in synergy with the NMDA antagonist MK-801.28 We have previously used a rabbit model of spinal cord ischemia to investigate the role of GABA in central nervous system ischemia. Using a "functional assay" of outcome following ischemia (recovery from paraplegia), we demonstrated that pharmacologic activation of the GABA-A receptor improves outcome following ischemia, while pharmacologic inhibition of this receptor worsens outcome from this injury. ${ }^{4}$ Vigabatrin has been shown to be protective in global ischemia models, ${ }^{13,23}$ when given prior to ischemia. Topiramate has also shown suggestive benefit in global ischemia models. ${ }^{29,30}$ In addition, Yang et al. reported benefit in a rodent model of focal ischemia. ${ }^{31}$

Despite the suggestive efficacy of these and other GABA activators for cerebral ischemia, however, this mouse model of focal ischemia failed to demonstrate neuroprotection by either vigabatrin or topiramate. A number of reasons may be postulated for this. These include failure of the model to reproduce the desired clinical state, failure of drug delivery, poor timing of drug delivery, inability of drug to produce the desired physiologic state, and failure of assessment tools to appreciate the desired outcome. 
The thread occlusion animal model is a well-established laboratory method for mimicking the focal cerebral ischemia associated with clinical stroke. ${ }^{32-35}$ This model has been used extensively at this laboratory, and has effectively demonstrated neuroprotection for a variety of other drugs. ${ }^{36-}$ 40 It has the advantages of providing reproducible infarct size for defined periods of reversible ischemia, and allows variation in infarct size by varying the duration of ischemia. ${ }^{8}$ Both histological and functional outcome measures are readily attainable. Infarcts induced by this study's methodology were both relatively small in the topiramate group (45 minute ischemic duration), and relatively large in the vigabatrin group (120 minute ischemic duration). The substantial difference in infarct sizes resulting from these two ischemic exposures provides further evidence for the model's sensitivity through histological measures. The variation in infarct sizes induced by these techniques also guards against the possibility that the neuroprotection provided by GABA potentiators has either an upper or lower limit of ischemic severity for effectiveness.

Seemingly, adequate doses of drugs were given to investigational animals by intraperitoneal injection in these studies. Vigabatrin and topiramate have different effects on GABA physiology, but each has been demonstrated in other models to effectively raise tissue GABA levels in brain. Vigabatrin reaches peak serum levels approximately one hour after administration. ${ }^{41}$ Brain GABA concentration rises rapidly, with 5-fold increases within 2 hours of administration of $1500 \mathrm{mg} / \mathrm{kg}$ in mice, which are sustained for over 24 hours. ${ }^{42}$ Doses of 80 to $1000 \mathrm{mg} / \mathrm{kg}$ are effective at aborting seizures in mice. ${ }^{43-45}$ Therefore, the two doses of 500 and $1000 \mathrm{mg} / \mathrm{kg}$ of vigabatrin used in this study, given two hours prior to the ischemic injury, appear to be reasonable to evaluate the hypothesis of neuroprotective benefit. Similarly, topiramate is effective after intraperitoneal injection in mouse seizure models at doses of 20 to $80 \mathrm{mg} / \mathrm{kg},{ }^{46-49}$ supplying reasonable expectations of neuroprotective effect at the 40 and $100 \mathrm{mg} / \mathrm{kg}$ doses used in this investigation. A prior study exploring topiramate as a neuroprotective drug for global cerebral ischemia demonstrated benefit at doses of $20 \mathrm{mg} / \mathrm{kg}$ in rodents. ${ }^{30}$ Despite the two seemingly reasonable doses of each drug, it is possible that the optimal dosing was still not used for one or both drugs for the induced cerebral ischemic conditions. Drugs may have unanticipated actions during pathophysiological states, including a bimodal response curve which confounds dose escalation assessment. Also, other effects of the drugs beyond that involving GABA potentiation may blunt their potential neuroprotection.

It is possible that pharmacologically appropriate doses of experimental drug were given, but rising brain levels of drug occurred at a time that was suboptimal for influencing ischemic pathophysiology. This is unlikely to be true for vigabatrin, since this drug was dosed prior to the induction of ischemia, at an interval that other animal models suggest would result in optimal brain levels of drug. This argument could be advanced for the topiramate group, since this drug was dosed at the time of reperfusion, and it is possible that pretreatment of drug would have resulted in greater retardation of ischemic injury. Even if this were true, however, a drug that requires pretreatment before an ischemic event to achieve a therapeutic response would have little clinical application. It is also possible that the ischemic environment itself also altered the potential effects of these drugs on GABA metabolism. Extracellular GABA concentrations may be elevated by tissue ischemia to the point that further potentiation of GABA activity by these drugs, or other GABA activators, may have little therapeutic effect.

\section{CONCLUSION}

Using this well-established animal model of reversible focal cerebral ischemia, neuroprotection could not be demonstrated for two drugs that potentiate the action of GABA in brain. Neither histological nor functional outcome measures were improved, despite both pre- and post-ischemic administration of drug in doses that are effective for other rodent cerebral pathophysiological states. Despite prior studies that suggest potential benefit of these and other potentiators, this study does not support a pharmacotherapeutic effect of GABA manipulation in ameliorating cerebral ischemic injury.

\section{REFERENCES}

1. Madden KP. Thrombolytic therapy for stroke. Mfld Clin J 2002;2:39-52.

2. Madden K. Optimal timing of thrombolytic therapy in acute ischaemic stroke. CNS Drugs 2002;16:213-218.

3. Madden K. NMDA receptor antagonists and glycine site NMDA antagonists. Curr Med Res Opin 2002;18 Suppl 2:s27-31.

4. Madden KP. Effect of gamma-aminobutyric acid modulation on neuronal ischemia in rabbits. Stroke 1994;25:2271-2274; discussion 2274-2275.

5. Glauser TA. Topiramate. Epilepsia 1999;40 Suppl 5:S71-80.

6. Mumford JP, Lewis PJ. Vigabatrin. Epilepsy Res Suppl 1991;3:161-168

7. Koizumi J, Yoshida Y, Nakazawa T, Ooneda G. Experimental studies of ischemic brain edema, I; a new experimental model of cerebral embolism in rats in which recirculation can be induced in the ischemic area. Jpn J Stroke 1986;8:1-8.

8. Clark WM, Lessov NS, Dixon MP, Eckenstein F. Monofilament intraluminal middle cerebral artery occlusion in the mouse. Neurol Res 1997;19:641-648.

9. Rothman SM, Olney JW. Glutamate and the pathophysiology of hypoxic-ischemic brain damage. Ann Neurol 1986;19:105111.

10. Albers GW, Goldberg MP, Choi DW. N-methyl-D-aspartate antagonists: ready for clinical trials in brain ischemia? Ann Neurol 1989;25:398-403.

11. Riveros N, Orrego F. N-methylaspartate-activated calcium channels in rat brain cortex slices. Effect of calcium channel blockers and of inhibitory and depressant substances. Neuroscience 1986;17:541-546.

12. Kelly PA, McCulloch J. The effects of the GABAergic agonist muscimol upon the relationship between local cerebral blood flow and glucose utilization. Brain Res 1983;258:338-342.

13. Abel MS, McCandless DW. Elevated gamma-aminobutyric acid levels attenuate the metabolic response to bilateral ischemia. J Neurochem 1992;58:740-744. 
14. Matsumoto K, Ueda S, Hashimoto T, Kuriyama K. Ischemic neuronal injury in the rat hippocampus following transient forebrain ischemia: evaluation using in vivo microdialysis. Brain Res 1991;543:236-242.

15. Tecoma ES, Choi DW. GABAergic neocortical neurons are resistant to NMDA receptor-mediated injury. Neurology 1989;39:676-682.

16. Gonzales C, Lin RC, Chesselet MF. Relative sparing of GABAergic interneurons in the striatum of gerbils with ischemia-induced lesions. Neurosci Lett 1992;135:53-58.

17. Saji M, Reis DJ. Delayed transneuronal death of substantia nigra neurons prevented by gamma-aminobutyric acid agonist. Science 1987;235:66-69.

18. Globus MY, Busto R, Martinez E, Valdes I, Dietrich WD, Ginsberg MD. Comparative effect of transient global ischemia on extracellular levels of glutamate, glycine, and gamma-aminobutyric acid in vulnerable and nonvulnerable brain regions in the rat. J Neurochem 1991;57:470-478.

19. Nilsson GE, Lutz PL. Release of inhibitory neurotransmitters in response to anoxia in turtle brain. Am J Physiol 1991;261:R32-37.

20. Alborch E, Torregrosa G, Terrasa JC, Estrada C. GABA receptors mediate cerebral vasodilation in the unanesthetized goat. Brain Res 1984;321:103-110.

21. Cross AJ, Jones JA, Baldwin HA, Green AR. Neuroprotective activity of chlormethiazole following transient forebrain ischaemia in the gerbil. Br J Pharmacol 1991;104:406-411.

22. Johansen FF, Diemer NH. Enhancement of GABA neurotransmission after cerebral ischemia in the rat reduces loss of hippocampal CA1 pyramidal cells. Acta Neurol Scand 1991;84:1-6.

23. Shuaib A, Ijaz S, Hasan S, Kalra J. Gamma-vinyl GABA prevents hippocampal and substantia nigra reticulata damage in repetitive transient forebrain ischemia. Brain Res 1992;590:13-17.

24. Sternau LL, Lust WD, Ricci AJ, Ratcheson R. Role for gammaaminobutyric acid in selective vulnerability in gerbils. Stroke 1989;20:281-287.

25. Hao JX, Xu XJ, Yu YX, Seiger A, Wiesenfeld-Hallin Z. Baclofen reverses the hypersensitivity of dorsal horn wide dynamic range neurons to mechanical stimulation after transient spinal cord ischemia; implications for a tonic GABAergic inhibitory control of myelinated fiber input. J Neurophysiol 1992;68:392-396.

26. Rosenbaum DM, Grotta JC, Pettigrew LC, Ostrow P, Strong R, Rhoades H, Picone CM, Grotta AT. Baclofen does not protect against cerebral ischemia in rats. Stroke 1990;21: 138-140.

27. Lyden PD, Hedges B. Protective effect of synaptic inhibition during cerebral ischemia in rats and rabbits. Stroke 1992;23:1463-1469.

28. Lyden PD, Lonzo L. Combination therapy protects ischemic brain in rats. A glutamate antagonist plus a gamma-aminobutyric acid agonist. Stroke 1994;25:189-196.

29. Lee S, Kim S, Kim J. Protective effect of topiramate against hippocampal neuronal damage after global ischemia in the gerbils. Neurosci Lett 2000;281:183-186.

30. Edmonds HL Jr, Jiang YD, Zhang PY, Shank R. Topiramate as a neuroprotectant in a rat model of global ischemia-induced neurodegeneration. Life Sci 2001;69:2265-2277.

31. Yang Y, Shuaib A, Li Q, Siddiqui MM. Neuroprotection by delayed administration of topiramate in a rat model of middle cerebral artery embolization. Brain Res 1998;804:169-176.

32. Yang G, Chan PH, Chen J, Carlson E, Chen SF, Weinstein P, Epstein CJ, Kamii H. Human copper-zinc superoxide dismutase transgenic mice are highly resistant to reperfusion injury after focal cerebral ischemia. Stroke 1994;25:165-170.

33. Soriano SG, Wang YF, Lipton SA, Dikkes P, Gutierrez-Ramos JC, Hickey PR. ICAM-1 dependent pathway is not involved in the development of neuronal apoptosis after transient focal cerebral ischemia. Brain Res 1998;780:337-341.
34. Li Y, Chopp M, Jiang N, Zaloga C. In situ detection of DNA fragmentation after focal cerebral ischemia in mice. Brain Res Mol Brain Res Jan 1995;28:164-168.

35. Connolly ES Jr, Winfree CJ, Stern DM, Solomon RA, Pinsky DJ. Procedural and strain-related variables significantly affect outcome in a murine model of focal cerebral ischemia. Neurosurgery 1996;38:523-531; discussion 532.

36. Clark WM, Rinker LG, Lessov NS, Lowery SL, Cipolla MJ. Efficacy of antioxidant therapies in transient focal ischemia in mice. Stroke 2001;32:1000-1004.

37. Clark WM, Rinker LG, Lessov NS, Hazel K, Hill JK, StenzelPoore M, Eckenstein F. Lack of interleukin-6 expression is not protective against focal central nervous system ischemia. Stroke 2000;31:1715-1720.

38. Clark WM, Rinker LG, Lessov NS, Hazel K, Eckenstein F. Time course of IL-6 expression in experimental CNS ischemia. Neurol Res 1999;21:287-292.

39. Clark WM, Lessov N, Lauten JD, Hazel K. Doxycycline treatment reduces ischemic brain damage in transient middle cerebral artery occlusion in the rat. J Mol Neurosci 1997;9:103-108.

40. Cipolla MJ, Lessov N, Clark WM, Haley EC Jr. Postischemic attenuation of cerebral artery reactivity is increased in the presence of tissue plasminogen activator. Stroke 2000;31:940-945.

41. Rey E, Pons G, Olive G. Vigabatrin. Clinical pharmacokinetics. Clin Pharmacokinet 1992;23:267-278.

42. Jung MJ, Lippert B, Metcalf BW, Bohlen P, Schechter PJ. Gamma-Vinyl GABA (4-amino-hex-5-enoic acid), a new selective irreversible inhibitor of GABA-T: effects on brain GABA metabolism in mice. J Neurochem 1977;29:797-802.

43. Holland KD, McKeon AC, Canney DJ, Covey DF, Ferrendelli JA. Relative anticonvulsant effects of GABAmimetic and GABA modulatory agents. Epilepsia 1992;33:981-986.

44. Sarhan S, Casara P, Knodgen B, Seiler N. (4S)-4-amino-5,6heptadienoic acid (MDL 72483): a potent anticonvulsant GABA-T inhibitor. Neurochem Res 1991;16:285-293.

45. Sayin U, Cengiz S, Altug T. Vigabatrin as an anticonvulsant against pentylenetetrazol seizures. Pharmacol Res 1993;28:325-331.

46. White HS, Brown SD, Woodhead JH, Skeen GA, Wolf HH. Topiramate modulates GABA-evoked currents in murine cortical neurons by a nonbenzodiazepine mechanism. Epilepsia 2000;41 Suppl 1:S17-20.

47. Wauquier A, Zhou S. Topiramate: a potent anticonvulsant in the amygdala-kindled rat. Epilepsy Res 1996;24:73-77.

48. Nakamura J, Tamura S, Kanda T, Ishii A, Ishihara K, Serikawa T, Yamada J, Sasa M. Inhibition by topiramate of seizures in spontaneously epileptic rats and DBA/2 mice. Eur J Pharmacol 1994;254(1-2):83-89.

49. Edmonds HL, Jr., Jiang YD, Zhang PY, Shank RP. Anticonvulsant activity of topiramate and phenytoin in a rat model of ischemia-induced epilepsy. Life Sci 1996;59:PL127-131.

$124 \quad C M \& R \quad 2003: 1$ (April) Madden et al.

